\title{
COMPARING ALMOST CONTINUOUS FUNCTIONS
}

\author{
PAUL E. LONG AND DONALD A. CARNAHAN
}

\begin{abstract}
Three definitions as to when a function from one topological space to another is called almost continuous are cited from the literature. Similarities and dissimilarities of two of these definitions are discussed.
\end{abstract}

Introduction. Recent mathematical literature contains three different definitions as to when a function from one topological space to another is called almost continuous. These definitions are as follows:

Definition 1. The function $f: X \rightarrow Y$ is almost continuous in the sense of Stallings [5] if given any open set $W \subset X \times Y$ containing the graph of $f$, there exists a continuous function $g: X \rightarrow Y$ such that the graph of $g$ is a subset of $W$.

Definition 2. The function $f: X \rightarrow Y$ is almost continuous at $x \in X$ in the sense of Husain [2] if for each open set $V \subset Y$ containing $f(x)$, the closure of $f^{-1}(V)$ is a neighborhood of $x$. If $f$ is almost continuous at each point of $X$, then $f$ is called almost continuous.

Definition 3. The function $f: X \rightarrow Y$ is called almost continuous at $x \in X$ in the sense of Singal and Singal [4] if for each open set $V \subset Y$ containing $f(x)$, there exists an open set $U \subset X$ containing $x$ such that $f(U)$ is a subset of the interior of the closure of $V$. If $f$ is almost continuous at each point of $X$, then $f$ is called almost continuous.

Of course, any continuous function satisfies all three of these definitions, but functions satisfying any one of the definitions need not be continuous as examples readily show. Examples also show the independence of these definitions. Example 1 of [3] shows that an almost continuous function in the sense of Husain need not be almost continuous in the sense of Singal and Singal. Example 2.1 of [4] shows that an almost continuous function in the sense of Singal and Singal need not be almost continuous in the sense of Husain. Consequently, Definitions 2 and 3 are completely independent of each other. Likewise, Examples 1 and 2 of [3] show that Definitions 1 and 2 are completely independent of each other. Example 2 of [3], $f: R \rightarrow R$ given by $f(x)=\sin (1 / x), x \neq 0$, and $f(0)=0$, is an example of a function

Received by the editors May 22, 1972.

AMS (MOS) subject classifications (1970). Primary 54C10.

Key words and phrases. Almost continuous function, noncontinuous function.

(c) American Mathematical Society 1973 
that is almost continuous in the sense of Stallings, but is not almost continuous in the sense of Singal and Singal. We leave as an open question, however, whether an almost continuous function in the sense of Singal and Singal is also almost continuous in the sense of Stallings.

Our primary concern in this paper is with the similarities and dissimilarities of Definitions 2 and 3. As a consequence, we use the abbreviation "a.c.H." to mean almost continuous in the sense of Husain and "a.c.S." to mean almost continuous in the sense of Singal and Singal. Also, $\mathrm{Cl}(A)$ will hence forth mean the closure of $A$ and $\operatorname{Int}(A)$ will mean the interior of A.

Results. Our first two theorems point out similarities between a.c.S. and a.c.H. functions.

THEOREM 1. Let $f: X \rightarrow Y$ be a function and let $g: X \rightarrow X \times Y$, given by $g(x)=(x, f(x))$, be its graph map. Then $g: X \rightarrow X \times Y$ is a.c.S. if and only if $f: X \rightarrow Y$ is a.c.S.

Proof. First, suppose $f$ is a.c.S. and we shall show $g$ is a.c.S. at an arbitrary point $x \in X$. Let $W \subset X \times Y$ be an open set containing the point $g(x)=$ $(x, f(x))$. Then there exist open sets $R \subset X, V \subset Y$ containing $x$ and $f(x)$, respectively, such that $R \times V \subset W$. Since $f$ is a.c.S., there exists an open set $U \subset R \subset X$ containing $x$ such that $f(U) \subset \operatorname{Int}(\mathrm{Cl}(V))$. Now observe that $U \times \operatorname{Int}(\mathrm{Cl}(V)) \subset \mathrm{Cl}(U) \times \mathrm{Cl}(V)=\mathrm{Cl}(U \times V)$ so that $\operatorname{Int}(U \times \operatorname{Int}(\mathrm{Cl}(V))) \subset$ $\operatorname{Int}(\mathrm{Cl}(U \times V))$. However, $\operatorname{Int}(U \times \operatorname{Int}(\mathrm{Cl}(V)))=\operatorname{Int}(U) \times \operatorname{Int}(\operatorname{Int}(\mathrm{Cl}(V)))=$ $U \times \operatorname{Int}(\mathrm{Cl}(V))$ since $U$ and $\operatorname{Int}(\mathrm{Cl}(V))$ are both open sets. Therefore, $U \times \operatorname{Int}(\mathrm{Cl}(V)) \subset \operatorname{Int}(\mathrm{Cl}(U \times V))$. In view of the fact that $f(U) \subset \operatorname{Int}(\mathrm{Cl}(V))$, we see that $g(U) \subset U \times \operatorname{Int}(\mathrm{Cl}(V)) \subset \operatorname{Int}(\mathrm{Cl}(U \times V)) \subset \operatorname{Int}(\mathrm{Cl}(W))$, showing that $g$ is a.c.S. at $x \in X$.

Conversely, suppose $g$ is a.c.S. Let $x \in X$ and let $V \subset Y$ be any open set containing $f(x)$. Then $X \times V$ is an open set in $X \times Y$ containing $(x, f(x))$ and, since $g$ is a.c.S., there exists an open set $U \subset X$ containing $x$ such that $g(U) \subset \operatorname{Int}(\mathrm{Cl}(X \times V))=\operatorname{Int}(X \times \mathrm{Cl}(V))=X \times \operatorname{Int}(\mathrm{Cl}(V))$. Thus, if $z \in U$, then $g(z)=(z, f(z)) \in X \times \operatorname{Int}(\mathrm{Cl}(V))$ so that $f(z) \in \operatorname{Int}(\mathrm{Cl}(V))$. Consequently, $f(U) \subset \operatorname{Int}(\mathrm{Cl}(V))$ which shows $f$ is a.c.S. at the point $x$.

TheOREM 2. Let $f: X \rightarrow Y$ be a function and let $g: X \rightarrow X \times Y$, given by $g(x)=(x, f(x))$, be its graph map. Then $g: X \rightarrow X \times Y$ is a.c.H. if and only if $f: X \rightarrow Y$ is a.c.H.

Proof. Let $f$ be a.c.H. We show $g$ is a.c.H. at the point $x \in X$. To do this, let $W \subset X \times Y$ be any open set containing $g(x)=(x, f(x))$. Then there exist open sets $U \subset X$ and $V \subset Y$ such that $x \in U, f(x) \in V$ and $U \times V \subset W$. Now note that $g^{-1}(U \times V)=U \cap f^{-1}(V)$. Since $f$ is a.c.H., we use Theorem 1 
of [3] to obtain the existence of an open set $S$ containing $x$ such that $S \subset U$ and $f^{-1}(V)$ is dense in $S$. Thus, $g^{-1}(U \times V)=U \cap f^{-1}(V) \supset S \cap f^{-1}(V)$ so that $\mathrm{Cl}\left(g^{-1}(U \times V)\right) \supset \mathrm{Cl}\left(S \cap f^{-1}(V)\right) \supset S$. The last inclusion follows from the fact that $S$ is open and $S \subset \mathrm{Cl}\left(f^{-1}(V)\right)$. It follows that $\mathrm{Cl}\left(g^{-1}(W)\right)$ is a neighborhood of $x$ implying $g$ is a.c.H. at the point $x$.

Conversely, let $g$ be a.c.H. and let $x \in X$. Consider any open set $V \subset Y$ containing $f(x)$. Then $X \times V$ is an open set in $X \times Y$ containing $(x, f(x))$, and since $g$ is a.c.H., $\mathrm{Cl}\left(g^{-1}(X \times V)\right)=\mathrm{Cl}\left(X \cap f^{-1}(V)\right)=\mathrm{Cl}\left(f^{-1}(V)\right)$ contains a neighborhood of $x$. Thus $f$ is a.c.H. at the point $x$.

We now note a common property between continuous retractions and a.c.S. retractions. By an a.c.S. retraction we mean an a.c.S. function $f: X \rightarrow A$ where $A \subset X$ and $f(a)=a$ for every $a \in A$. We call $A$ an a.c.S. retract of $X$.

THEOREM 3. Let $A \subset X$ and let $f: X \rightarrow A$ be an a.c.S. retraction of $X$ onto $A$. If $X$ is Hausdorff, then $A$ is a closed subset of $X$.

Proof. Suppose $A$ is not closed. Then there exists a point $x \in X$ such that $x \in \mathrm{Cl}(A)$ but $x \notin A$. It follows that $f(x) \neq x$ because $f$ is an a.c.S. retraction of $X$ onto $A$. Since $X$ is Hausdorff, there exist open sets $U$ and $V$ in $X$ such that $x \in U, f(x) \in V$ and $U \cap V=\varnothing$. Thus, $U \cap \operatorname{Int}(\mathrm{Cl}(V))=\varnothing$. Now let $W$ be any open set in $X$ containing $x$. Then $U \cap W \neq \varnothing$ is open and does contain $x$. Consequently $(U \cap W) \cap A \neq \varnothing$ and, in fact, contains infinitely many points of $A$. Let $z \in(U \cap W) \cap A$. Then $f(z)=z$ so that $z \notin \operatorname{Int}(\mathrm{Cl}(V))$ because $U \cap \operatorname{Int}(\mathrm{Cl}(V))=\varnothing$. It follows that $f(W) \notin$ $\operatorname{Int}(\mathrm{Cl}(V))$ which implies that $f$ is not a.c.S. at the point $x$. This contradiction to our hypothesis means $A$ must be closed.

The a.c.H. retractions do not share this same property, however. The following example is of an a.c.H. retraction of a Hausdorff space onto a retract that is not closed.

EXAMPLE 1. Let $I$ be the closed unit interval with the standard subspace topology from the reals. Let $Q$ represent the rationals belonging to $I$. Let $f: I \rightarrow Q$ be defined as follows: $f(x)=x$ if $x \in Q$ and $f(x)=0$ if $x \in I-Q$. Then $f$ is an a.c.H. retraction of $I$ onto $Q, I$ is Hausdorff, but $Q$ is not closed in $I$.

We next investigate when our functions preserve connected sets. To do so we use the definition of a regularly-open set [4]. A set is regularly-open if it is the interior of its own closure. For a.c.S. functions we have the following theorem:

THEOREM 4. Let $f: X \rightarrow Y$ be an a.c.S. surjection. If $X$ is connected, then $f(X)=Y$ is connected. 
Proof. Suppose $X$ is connected and that $f(X)=Y=A \cup B$ where $A$ and $B$ are separated. Then both $A$ and $B$ are closed as well as open, hence are regularly open subsets of $Y$. Since $f$ is a.c.S., $f^{-1}(A)$ and $f^{-1}(B)$ are open in $X$ by Theorem 2.2(b) of [4]. Also, $f^{-1}(A) \cap f^{-1}(B)=\varnothing$ because $A \cap B=\varnothing$, and $f^{-1}(A) \cup f^{-1}(B)=X$ due to the fact that $Y=A \cup B$. It follows that the sets $f^{-1}(A)$ and $f^{-1}(B)$ give a separation of $X$ and this contradicts the hypothesis that $X$ is connected. Therefore $Y$ must be connected.

Functions of the a.c.S. type do not preserve connected sets in general, however. The reason is that even though a.c.S. functions may be restricted in their domain [4, Theorem 2.6], they may not, in general, be restricted in the range space to $f \mid A: A \rightarrow f(A)$ where $f(A)$ has the subspace topology. Our next example shows this with an a.c.S. function $f: X \rightarrow Y$ such that $f: X \rightarrow f(X)$ is not a.c.S.

EXAmple 2. Let $X=Y=\{a, b, c\}$. Let the topology on $X$ be indiscrete and topologize $Y$ with $\{\varnothing, Y,\{a, c\},\{b, c\},\{c\}\}$. Let $f: X \rightarrow Y$ be given by $f(a)=a, f(b)=f(c)=b$. Then $f$ is a.c.S. and the subspace $f(X)=\{a, b\}$ of $Y$ has the discrete topology. Thus, both $\{a\}$ and $\{b\}$ are open in the subspace topology giving immediately that $f: X \rightarrow f(X)$ is not a.c.S.

Functions of the a.c.H. type do not behave even as well as a.c.S. functions when preserving connected sets is concerned. Example 1 of [3] shows a.c.H. functions need not preserve connected sets and the next example shows that, unlike a.c.S. functions, a.c.H. surjections need not preserve connected sets either.

ExAmple 3. Let $X=Y=\{0,1\}$ where $X$ has the indiscrete topology and $Y$ has the discrete topology. Then the identity function $i: X \rightarrow Y$ is a.c.H., $X$ is connected, but $i(X)=Y$ is not connected.

In two of the theorems that follow we use the following fact:

LEMMA. Let $f: X \rightarrow Y$ be an open function. Then for every subset $B \subset Y$, $f^{-1}(\mathrm{Cl}(B)) \subset \mathrm{Cl}\left(f^{-1}(B)\right)$.

Proof. This is a direct consequence of Theorem 11.2, Chapter III of [1].

The first of these theorems gives a condition as to when a.c.S. functions are also a.c.H. functions.

THEOREM 5. Let $f: X \rightarrow Y$ be an open a.c.S. function. Then $f$ is a.c.H.

Proof. Let $x \in X$ and let $V \subset Y$ be an open set containing $f(x)$. By the Lemma, $f^{-1}(\mathrm{Cl}(V)) \subset \mathrm{Cl}\left(f^{-1}(V)\right)$. Now we observe that $\operatorname{Int}(\mathrm{Cl}(V))$ is a regularly-open set and that $V \subset \operatorname{Int}(\mathrm{Cl}(V)) \subset \mathrm{Cl}(V)$. Since $f$ is a.c.S., $f^{-1}(\operatorname{Int}(\mathrm{Cl}(V)))$ is open in $X$ by Theorem 2.2 of [4]. Thus, $f^{-1}(\operatorname{Int}(\mathrm{Cl}(V))) \subset$ $f^{-1}(\mathrm{Cl}(V)) \subset \mathrm{Cl}\left(f^{-1}(V)\right)$ and consequently $\mathrm{Cl}\left(f^{-1}(V)\right)$ is a neighborhood of $x$. By Definition $2, f$ is a.c.H. 
Corollary. If $f: X \rightarrow Y$ is an a.c.S. bijection, $X$ compact and $Y$ Urysohn, then $f$ is a.c.H.

Proof. Corollary 2.2 of [4].

EXAmple 4. An a.c.S. open function need not be continuous. Let $X=\{a, b, c\}$ with topology $\{\varnothing, X,\{b, c\}\}$ and let $Y$ be the Sierpiński space. Then $f: X \rightarrow Y$ given by $f(a)=f(b)=0$ and $f(c)=1$ is a.c.S. but not continuous.

THEOREM 6. Let $f: X \rightarrow Y$ be a.c.S. and let $V \subset Y$ be open. If $x \notin f^{-1}(V)$ but $x \in \mathrm{Cl}\left(f^{-1}(V)\right)$, then $f(x) \in \mathrm{Cl}(V)$.

Proof. Let $x \in X$ be such that $x \notin f^{-1}(V)$ but $x \in \mathrm{Cl}\left(f^{-1}(V)\right)$ and suppose $f(x) \notin \mathrm{Cl}(V)$. Then there exists an open set $W$ such that $f(x) \in W$ and $W \cap V=\varnothing$. Thus, $\mathrm{Cl}(W) \cap V=\varnothing$ and $\operatorname{Int}(\mathrm{Cl}(W)) \cap V=\varnothing$. Since $f$ is a.c.S., there exists an open set $U \subset X$ such that $x \in U$ and $f(U) \subset \operatorname{Int}(\mathrm{Cl}(W))$. As a consequence, $f(U) \cap V=\varnothing$. However, since $x \in \mathrm{Cl}\left(f^{-1}(V)\right), U \cap$ $f^{-1}(V) \neq \varnothing$ so that $f(U) \cap V \neq \varnothing$. We have a contradiction. It follows that $f(x) \in \mathrm{Cl}(V)$.

THEOREM 7. Let $f: X \rightarrow Y$ be an open a.c.S. function. Then for each open $V \subset Y, \mathrm{Cl}\left(f^{-1}(V)\right) \subset f^{-1}(\mathrm{Cl}(V))$.

Proof. Let $V \subset Y$ be open. By Theorem $6, f\left(\mathrm{Cl}\left(f^{-1}(V)\right)\right) \subset \mathrm{Cl}(V)$. Since $\mathrm{Cl}\left(f^{-1}(V)\right) \subset f^{-1}\left(f\left(\mathrm{Cl}\left(f^{-1}(V)\right)\right)\right)$ for any function, we have

$$
\mathrm{Cl}\left(f^{-1}(V)\right) \subset f^{-1}\left(f\left(\mathrm{Cl}\left(f^{-1}(V)\right)\right)\right) \subset f^{-1}(\mathrm{Cl}(V)) .
$$

COROLlaRY.Let $f: X \rightarrow Y$ be an open a.c.S. function. Then for each open $V \subset Y, \mathrm{Cl}\left(f^{-1}(V)\right)=f^{-1}(\mathrm{Cl}(V))$.

Proof. Theorem 7 and the Lemma.

THEOREM 8. An open a.c.H. function $f: X \rightarrow Y$ is a.c.S. if and only if $\mathrm{Cl}\left(f^{-1}(V)\right)=f^{-1}(\mathrm{Cl}(V))$ for every open $V \subset Y$.

Proof. If $f$ is a.c.S., then the Corollary to Theorem 7 gives the condition.

Conversely, if $f$ is a.c.H., open and has the condition, we show $f$ is a.c.S. Let $x \in X$ and let $V \subset Y$ be open containing $f(x)$. Since $f$ is a.c.H., there exists an open set $U \subset X$ such that $x \in U \subset \mathrm{Cl}\left(f^{-1}(V)\right) \subset f^{-1}(\mathrm{Cl}(V))$. It follows that $f(U) \subset f\left(f^{-1}(\mathrm{Cl}(V))\right) \subset \mathrm{Cl}(V)$. By Definition 2.3 of [4], $f$ is weakly continuous, and since $f$ is open, $f$ is a.c.S. by Theorem 2.3 of [4].

\section{REFERENCES}

1. James Dugundji, Topology, Allyn and Bacon, Boston, Mass., 1966. MR 33 \#1824.

2. T. Husain, Almost continuous mappings, Prace Mat. 10 (1966), 1-7. MR 36 \#3322. 
3. Paul E. Long and Earl E. McGehee, Jr., Properties of almost continuous functions, Proc. Amer. Math. Soc. 24 (1970), 175-180. MR 40 \#4931.

4. M. K. Singal and Asha Rani Singal, Almost-continuous mappings, Yokohama Math. J. 16 (1968), 63-73. MR 41 \#6182.

5. J. R. Stallings, Fixed point theorems for connectivity maps, Fund. Math. 47 (1959), 249-263. MR 22 \#8485.

Department of Mathematics, University of Arkansas, Fayetteville, Arkansas 72701 